文章编号 : $1001-9081(2010) 01-0266-04$

\title{
基于事件的局部行为模型的合并
}

\author{
郭正虎，陈中育,张纪昌 \\ (浙江师范大学 数理与信息工程学院,浙江 金华 321004) \\ (guozhenghu@ foxmail. com)
}

\begin{abstract}
摘 要:采用模态迁移系统描述系统行为, 针对局部行为模型中存在的不确定行为,提出一种基于事件的局部行 为模型合并方法。该方法首先定义局部行为模型之间的精化关系, 利用精化关系产生合并规则,运用合并规则产生 行为模型的极小共同精化模型或最小共同精化模型, 从而消除局部行为模型中存在的不确定行为。最后通过一个示 例对该方法的有效性作出说明。
\end{abstract}

关键词: 模态迁移系统; 不确定行为;精化;合并规则

中图分类号: TP311.5 文献标志码: A

\section{Event-based merging of partial behavior models}

\author{
GUO Zheng-hu, CHEN Zhong-yu, ZHANG Ji-chang \\ (College of Mathematics, Physics and Information Engineering, Zhejiang Normal University, Jinhua Zhejiang 321004, China)
}

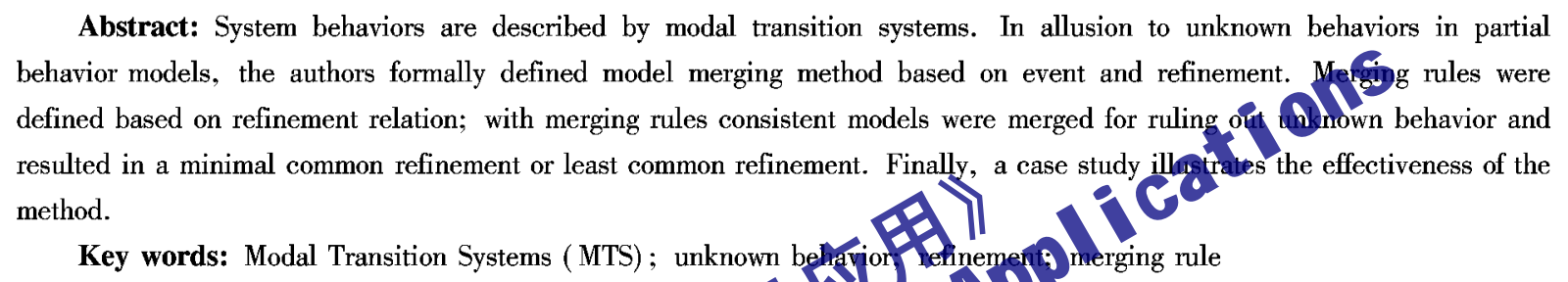

Key words: Modal Transition Systems (MTS); unknown behajor letnemen merging rule

0 引言

场景是一种分析与验证需求的有效工具, 因正基于场䑾0 的分析与设计受到广泛关注 ${ }^{[1]}$ 。一个系统需求由击个局部 场景构成,这些场景是由不同的相关人员根据白己所关注的 业务功能并结合自己的知识给出, 每介场景描述系统中不同 用户的需求, 多个场景所描述的用 10 需求可能存在某些业务 需求的重叠 ${ }^{[2]}$, 如何把从这些局部场景中抽取的行为模型合 并成一个完整的系统位为模型值得研究。文献 [1]使用标号 迁移系统 (Label Transition System, LTS) 从基于场景的规约中 合成行为模型, LTS 模型实现了系统行为建模和推理的形式 化,为各种自动分析技术提供理论基础。但 LTS 模型所描述 的行为必须是系统中的确定行为, 而在系统逐步详细描述过 程中局部场景可能含有不确定行为, LTS 模型的确定性假设 限制了在软件开发过程中对含有不确定行为的建模和分析, 因此本文希望对系统行为进行建模和分析过程中能够区分确 定行为和不确定行为, 并消除不可能行为。目前支持不确定 行为的形式化方法主要有混合迁移系统 ${ }^{[2]}$ 、多值 Kripke 结 构 $^{[3]}$ 和模态迁移系统 ${ }^{[4]}$ 等。

行为模型的并行复合主要针对不同构件系统的行为组 合, 而行为模型的合并是从同一构件系统的两个局部行为模 型中获得一个精化的模型。本文采用模态迁移系统对系统行 为进行建模, 在精化关系的基础之上结合隐藏操作, 给出共同 精化模型, 并根据精化程度定义了极小共同精化和最小共同 精化。本文在分析现有合并算法的基础上, 指出现有合并算 法的局限性, 即总是假设模型之间同一事件的源状态和目标
状态是一致的,针对此局限性, 文中提出了源状态一致标号集 和盲标一致标号集,在基于事件合并的同时结合事件的源状 态和目标状态给出了新的合并规则。运用合并规则可以产生 极小共同精化模型或最小共同精化模型。在对合并规则进行 解释说明之后又通过水泵控制系统对合并规则的有效性进行 说明。

\section{1 背景知识}

本章将介绍模态迁移系统及其精化等概念, 其中与行为 模型合并相关的一些概念主要参考文献 $[2,5]$ 。

标号迁移系统 (LTS) 用于分布式系统行为的形式化描 述,成为分布式系统规约、实现和测试研究的有效工具。LTS 是一个状态迁移的系统,其中迁移被标上了行为。LTS 的行 为集称为通信字母表,构成模型系统与环境的相互作用。此 外,LTS 可以含有不被环境观察的内部行为 $\tau$ 。

定义 1 标号迁移系统 LTS。假设 States 是一个状态集 合, $A c t$ 是一个可观测行为标号集合, $A c t_{\tau}=A c t \cup\{\tau\}$ 。一个标 号迁移系统是一个四元组 $P=\left(S, L, \Delta, s_{0}\right)$, 其中: $S \subseteq$ States 是一个有限状态集合; $L \subseteq A c t_{\tau}$ 是一个有限的标号集合; $\Delta \subseteq$ $(S \times L \times S)$ 是两个状态之间的转换关系; $s_{0} \in S$ 是初始状态。 文中使用 $\alpha P=L \backslash\{\tau\}$ 来定义 $P$ 的通信字母表。

标号迁移系统虽然可以描述系统行为, 但是不包含不确 定的行为。模态迁移系统 (Model Transition Systems, MTS) 能 明确描述出系统中的未知行为模型, 模态迁移系统是标号迁 移系统的扩展, 添加了系统中不确定的迁移关系集。

定义 2 模态迁移系统 MTS。一个模态迁移系统是五元

收稿日期:2009-07-19; 修回日期:2009-08-27。

作者简介: 郭正虎 $(1982-)$, 男, 河南濮阳人,硕士研究生,主要研究方向: 软件工程、形式化方法; 陈中育 $(1964-)$, 男,浙江浦江人,教 授,主要研究方向: 软件工程、形式化方法; 张纪昌 (1984-), 男, 河南开封人, 硕士研究生, 主要研究方向: 软件工程。 
组 $M=\left(S, L, \Delta^{r}, \Delta^{p}, s_{0}\right)$ 。其中: $\Delta^{r} \subseteq \Delta^{p} ;\left(S, L, \Delta^{r}, s_{0}\right)$ 表示系 统必然迁移的 LTS; $\left(S, L, \Delta^{p}, s_{0}\right)$ 表示系统的允许迁移 (但不 一定必然) 的 LTS。

文中使用 $\alpha M=L \backslash\{\tau\}$ 来定义 $M$ 的通信字母表。

$\Delta^{r}$ 表示必然迁移关系集, $\Delta^{p}$ 表示允许迁移关系集, 本文 使用 $\Delta^{p}-\Delta^{r}$ 表示可能迁移关系集。在行为模型图中可能性迁 移标号用问号来标记, 以便区分必然迁移 (在 $\Delta^{r}$ 中), LTS 是

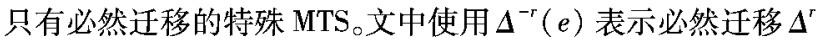
中事件 $e$ 的源状态, $\Delta^{r}(e)$ 表示必然迁移 $\Delta^{r}$ 中的目标状态, 同 样使用 $\Delta^{-p}(e)$ 表示允许迁移 $\Delta^{p}$ 中事件 $e$ 的源状态, $\Delta^{p}(e)$ 表 示允许迁移 $\Delta^{p}$ 中事件 $e$ 的目标状态。

给定一个 MTS, $M=\left(S, L, \Delta^{r}, \Delta^{p}, s_{0}\right)$, 使用 $M_{s} \stackrel{l}{\longrightarrow} M_{t}$ 表 示在模型 $M$ 中状态 $s$ 可以通过标号 $l$ 必然迁移到状态 $t$, 也就 是 $(s, l, t) \in \Delta^{r}$ 。同样 $M_{s} \stackrel{l}{\longrightarrow} M_{t}$ 表示表示状态 $s$ 可以通过标 号 $l$ 可能迁移到状态 $t$, 也就是 $(s, l, t) \in \Delta^{y}-\Delta^{r}$ 。

MTS 精化是从不确定的行为描述中获取一个确定的行 为描述, 可以理解为是两个不确定行为模型的更确定的关系。 直观上说, MTS 的精化是把行为模型中的可能迁移转化为必 然迁移,或者排除不必要的可能迁移。如果 MTS $N$ 保留了 $M$ 的所有必然和允许迁移, 或者说 $N$ 能模拟 $M$ 的必然迁移, $M$ 能 模拟 $N$ 的可能迁移,就说 $N$ 是 $M$ 的精化。

定义 3 精化。假设 $\mathscr{P}$ 是所有 MTS 的论域。 $N$ 是 $M$ 的一 个精化, 记为 $M \leq N$, 其中 $\alpha N=\alpha M$, 且 $(M, N)$ 包含的精化 关系 $R \subseteq \mathscr{P} \times \mathscr{P}$, 并且对于任意 $l \in A c t_{\tau}$ 满足如下规䞍

1) $\left(M \stackrel{l}{\longrightarrow} M^{\prime}\right) \Rightarrow\left(\exists N^{\prime} \cdot N \stackrel{l}{\longrightarrow} N^{\prime} \wedge(\right.$ H

2) $\left(N \stackrel{l}{\longrightarrow} N^{\prime}\right) \Rightarrow\left(\exists M^{\prime} \cdot M \stackrel{l}{\longrightarrow} M^{\prime} \wedge\left(M^{\prime}, N(0)\right.\right.$

注意: 条件 2) 保证如果 $N$ 有一个必然香移, $M$ 会有一个 可能迁移或者必然迁移, 但是如果 $N$ 有一个四能迁移, 那么 $M$ 一定是一个可能迁移; 否则就与条 $(9 f(1)$ 相违背。

图 1 中模型 $C$ 是模型 $A$ 约精化 $(A \leq C)$, 模型 $C$ 把 $A$ 中的 可能迁移 $(1, b ?, 2)$ 和 $(1, c ?, 3)$ 都精化为必然迁移。当然模型 $C_{1}$ 和 $C_{2}$ 也是 $A$ 的精化, 只不过在精化中选择了不同的可能迁 移标号进行精化。
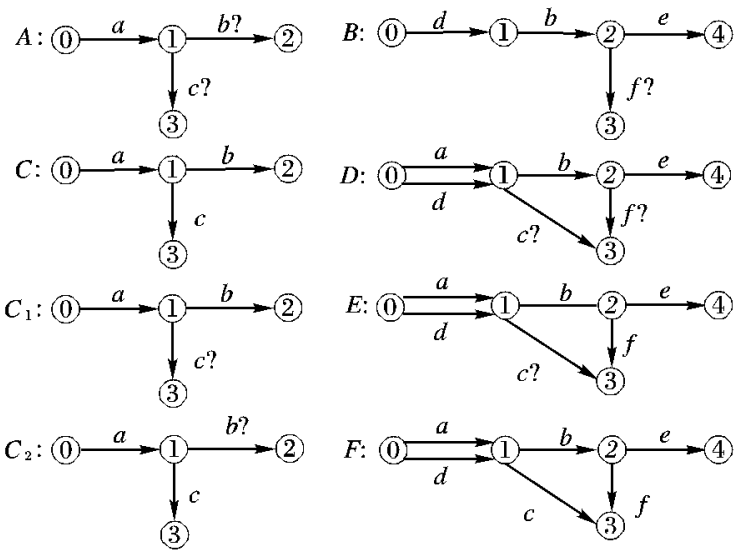

图 1 MTS 模型

尽管精化可以获取更加详细的模型描述, 但是要求有相 同的通信字母表。实际上模型的详细描述扩大了系统模型的 通信字母表, 来描述先前没有考虑到的系统行为。为了获取 模型细化的这一特性引人隐藏概念。

定义 4 隐藏。假设 $M=\left(S, L, \Delta^{r}, \Delta^{p}, s_{0}\right)$ 是一个 MTS,
$X \subseteq A c t$ 是一组可观察的动作, 隐藏动作 $X$ 的 $M$ 是 $\left(S, L \backslash X, \Delta^{r^{\prime}}\right.$, $\left.\Delta^{p^{\prime}}, s_{0}\right)$, 表示成 $M \backslash X$, 其中 $\Delta^{r^{\prime}}$ 和 $\Delta^{p^{\prime}}$ 是满足隐藏操作规则的最 小关系。使用 $M @ \alpha X$ 来定义 $M \backslash(A c t \backslash X)$ 。

$$
\begin{array}{cc}
\frac{M \stackrel{e}{\longrightarrow}}{\longrightarrow} M^{\prime} & e \notin X \\
(M \backslash X) \stackrel{e}{\longrightarrow}\left(M^{\prime} \backslash X\right) & \gamma \in\{r, m\} \\
\frac{e}{\longrightarrow} M^{\prime} & e \in X \\
(M \backslash X) \stackrel{\tau}{\longrightarrow}\left(M^{\prime} \backslash X\right) & \gamma \in\{r, m\}
\end{array}
$$

直观上看, 通过合并从同一系统的两个不确定描述中获 取更多信息,在这种情况下精化概念是在两个不确定行为模 型中获得更确定的关系。因此,合并同一个系统的两个模型 就是找到一个共有 (共同)精化,也就是找到一个比这两个模 型都更完整的模型。

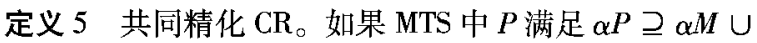
$\alpha N, M \leqq P @ \alpha M$ 和 $N \lesssim P @ \alpha N$, 那么 $P$ 就是 $\operatorname{MTS}$ 的 $M$ 和 $N$ 的 共同精化。

图 1 中模型 $D 、 E 、 F$ 都是 $A$ 和 $B$ 的共同精化, $E$ 和 $F$ 也是 $D$ 的一个精化。 $F$ 是其他共有精化的精化, 称 $D$ 是极小共同精 化。

定义 6 极小共同精化 LCR。假琪模态迁移系统 $P$ 是 $M$ 和 $N$ 的一个共同精化, 且 $\alpha P=\alpha M \cup \alpha N$, 如果对于 $M$ 和 $N$ 的 任一共精化 $Q$ 都满臬 $P \leq Q @ \alpha P$, 则称 $P$ 是 $M$ 和 $N$ 的极小 有精化。1001

由定义命以看出极小共同精化是独一无二的，使用 LCF 来表示 $M$ 和 $N$ 的极小共同精化。 $M$ 和 $N$ 的共同精化存 在并不能保证 $L C R_{M, N}$ 的存在,为此提出了最小共同精化的概 念。

定义 7 最小共同精化 $\mathrm{MCR}_{0}$ 如果模态迁移系统 $P$ 是 $M$ 和 $N$ 的一个最小共同精化,且 $\alpha P=\alpha M \cup \alpha N$, 那么一定不存 在另一个 $M$ 和 $N$ 共同精化 $Q \neq \equiv$ 使得 $Q @ \alpha P \leqslant P$ 。

由定义可以看出最小共同精化并不满足唯一性, 文中使 用 $M C R(M, N)$ 来表示 $M$ 和 $N$ 的最小共同精化集 ${ }_{0} M$ 和 $N$ 的 $L C R_{M, N}$ 是它们一个 MCR, 如果 $M$ 和 $N$ 的 MCR 是唯一的, 此 $\operatorname{MCR}$ 也是 $L C R_{M, N}$ 。

行为模型的合并实质就是求两个行为原有模型的 MCR 或者 LCR。

\section{2 合并算法}

尽管合并的算法已经有人提出,但是一个完整且普遍适 用的算法还没有提出, 本章首先分析两种不同的合并算法, 最 后提出一种新的算法,该算法可以对状态不一致的行为模型 进行合并。

\section{1 现有算法的局限性}

首先介绍文献[6]中提出的模型之间的连接操作。

定义 8 连接 Conjunction。MTS 中 $M$ 和 $N$ 的连接定义为 $M \wedge N=\left(S_{M} \times S_{N}, L, \Delta_{M \wedge N}^{r}, \Delta_{M \wedge N}^{p},\left(m_{0}, n_{0}\right)\right)$, 记为 $C J_{M, N}$, 其 中 $\Delta_{M \wedge N}^{r}, \Delta_{M \wedge N}^{p}$ 是满足下列规则的最小关系:

$$
\begin{array}{r}
R P: \frac{M \stackrel{l}{\longrightarrow} M^{\prime}, N \stackrel{l}{\longrightarrow} N_{p}^{\prime}}{(M, N) \stackrel{l}{\longrightarrow}\left(M^{\prime}, N^{\prime}\right)} \\
P R: \frac{M \stackrel{l}{\longrightarrow} M^{\prime}, N \stackrel{l}{\longrightarrow} N^{\prime}}{(M, N) \stackrel{l}{\longrightarrow}\left(M^{\prime}, N^{\prime}\right)}
\end{array}
$$




$$
P P: \frac{M \stackrel{l}{\longrightarrow} M_{p}^{\prime}, N \stackrel{l}{\longrightarrow} N_{p}^{\prime}}{(M, N) \stackrel{l}{\longrightarrow}\left(M^{\prime}, N^{\prime}\right)}
$$

连接的局限性就是有时 LCR 存在, 但是并没有给出结 果, 实际上在这种情况下, 这个运算就没有一个共同精化。

为了解决连接运算带来的问题, 文献 [4] 中提出了合并 运算, 文献 5 ] 又对这种算法进行了改进。并通过 $+_{u}$ 产生所 有最小共同精化的一个上界,通过 $+_{l}$ 运算产生所有最小共同 精化的一个下界, 当条件充足时 $+_{l}$ 产生的就是极小共同精 化。

无论是文献 $[6]$ 的连接运算还是文献 $[4,5]$ 的合并都是 假定模型的状态一致。也就是说, 同一个事件 $e$ 在两个模型 中源状态和目标状态总是假定是一致的, 这种合并只是基于 事件的合并。但是在实际中这种假设受到很大限制, 因此本 文提出了针对状态不一致的行为模型提出了新合并算法。

\section{2 新的合并算法}

由于新算法考虑到标号的源状态和目标状态是否一致, 为此给出源状态一致标号集和目标状态一致标号集两个定 义, 还给出了 MTS一致性定义, 因为只有满足一致性的 MTS 才能进行合并。

定义 9 源状态一致标号集。对于给定的 MTS 中 $M$ 和 $N, M$ 和 $N$ 的源状态一致标号集 $\zeta_{M, N}^{s r c}$ 满足以下条件,

1) $\forall e \in(\alpha M \cap \alpha N)$, 如果存在 $\Delta_{M}^{-\gamma}(e)=\Delta_{N}^{-\gamma}(e), \gamma \in$ $\{r, m, p\}$, 那么 $e \in \zeta_{M, N}^{s r c}$;

2) $e \in \zeta_{M, N}^{s r c}$, 一定满足 $\Delta_{M}^{-\gamma}(e)=\Delta_{N}^{-\gamma}(e), \gamma \in\{r$

定义 10 目标状态一致标号集。对于给定 和 $N, M$ 和 $N$ 的目标状态一致标号集 $\zeta_{M, N}^{\text {trg }}$ 满足 1) $\forall e \in(\alpha M \cap \alpha N)$, 如果存在 $\Delta_{M}^{\gamma}(e) \in \Delta_{N}^{\gamma}(e), \gamma \in$
$\{r, m, p\}$, 那么 $e \in \zeta_{M, N}^{i t g} ;$

2) $\forall e \in \zeta_{M, N}^{t r g}$, 一定满足 $\Delta_{M}^{\gamma}(e) \gamma=\Delta_{N}^{\gamma}(e), \gamma \in\{r, m, p\}$ 。

定义 11 一致性。给完MTS $M=\left(S_{M}, L_{M}, \Delta_{M}^{r}, \Delta_{M}^{p}, s_{0_{M}}\right)$ 和 $N=\left(S_{N}, L_{N}, \Delta_{N}^{r}, \Delta_{N}, s_{0_{N}}\right), \forall e \in \alpha M \cap \alpha N$ 如果 $\Delta_{M}^{-r}(e)=$ $\Delta_{N}^{-r}(e)$, 那么一定有 $\Delta_{M}(e)=\Delta_{N}^{r}(e)$, 就说 $M$ 和 $N$ 满足一致 性。

本文中所讨论的行为模型都要满足一致性。因为我们认 为从相同的源状态经过相同的标号 $e$ 应该迁移到达相同的目 标状态。

定义 12 迁移不确定状态。假设 $M=\left(S, L, \Delta^{r}, \Delta^{p}, s_{0}\right)$ 是 MTS, 对于状态 $s$ 如果 $M$ 中存在状态 $q$ 和 $r$, 使得 $M_{s} \stackrel{e}{\longrightarrow} M_{q}$ 和 $M_{s} \stackrel{e}{\longrightarrow} M_{r}$ 成立且 $M_{q} \neq M_{r}$, 就说 $M_{s}$ 在 $e$ 上的正移是不确 定的,状态 $s$ 是迁移不确定的状态。

MTS 之间的一致性保证两个行为模型中的两个必要迁 移 $\mathrm{e}$ 迁移到确定的同一状态, 而对于每一个行为模型同样要 确保不包含迁移不确定的状态。

下面介绍 $+_{c r}$ 运算, $M+{ }_{c r} N$ 的结果是 $M$ 和 $N$ 的一个极小 共同精化。

定义 $13+{ }_{c r}$ 运算。假设 $M$ 和 $N$ 是 MTS, 其中 $M=\left(S_{M}\right.$, $\left.L_{M}, \Delta_{M}^{r}, \Delta_{M}^{p}, s_{0_{M}}\right)$ 和 $N=\left(S_{N}, L_{N}, \Delta_{N}^{r}, \Delta_{N}^{p}, s_{0_{N}}\right), M+{ }_{c r} N$ 是 MTS $M=\left(S_{M} \times S_{N}, L_{M} \cup L_{N}, \Delta^{r}, \Delta^{p},\left(s_{0_{M}}, s_{0_{N}}\right)\right), \Delta^{r}$ 和 $\Delta^{p}$ 是满足 $+_{c r}$ 运算规则的最小关系。

$$
\begin{aligned}
& R D: \frac{M \stackrel{e}{\longrightarrow}{ }_{r} M^{\prime}}{M+{ }_{c r} N \stackrel{e}{\longrightarrow} M^{\prime}+{ }_{c r} N}, e \notin \alpha N \\
& D R: \frac{N \stackrel{e}{\longrightarrow} N_{r} N^{\prime}}{M+{ }_{c r} N \stackrel{e}{\longrightarrow} M+{ }_{c r} N^{\prime}}, e \notin \alpha M \\
& M D: \frac{M \stackrel{e}{\longrightarrow} M_{m} M^{\prime}}{M+{ }_{c r} N \stackrel{e}{\longrightarrow}{ }_{m} M^{\prime}+{ }_{c r} N}, e \notin \alpha N \\
& D M: \frac{N \stackrel{e}{\longrightarrow} N_{r}^{\prime}}{M+{ }_{c r} N \stackrel{e}{\longrightarrow} M+{ }_{c r} N^{\prime}}, e \notin \alpha M \\
& R M_{E E}: \frac{M \stackrel{e}{\longrightarrow} M^{\prime}, N \stackrel{e}{\longrightarrow} N_{m}^{\prime}}{M+{ }_{c r} N \stackrel{e}{\longrightarrow} M^{\prime}{ }^{\prime}{ }_{c r} N^{\prime}}, e \neq \tau \text { 且 } e \in \zeta_{M, N}^{s s c}, e \in \zeta_{M, N}^{\text {tre }} \\
& R M_{E D}: \frac{M \stackrel{e}{\longrightarrow} M_{r_{1}} M^{\prime}, N \stackrel{e}{\longrightarrow}{ }_{m_{1}} N^{\prime}}{M+{ }_{c r} N \stackrel{e}{\longrightarrow} M_{r}^{\prime}+{ }_{c r} N, M+{ }_{c r} N{\nrightarrow_{m_{1}}} M+{ }_{c r} N^{\prime}}, e \neq \tau \text { 且 } \\
& e \in \zeta_{M, N}^{s r c}, e \notin \zeta_{M, N}^{t r g}
\end{aligned}
$$

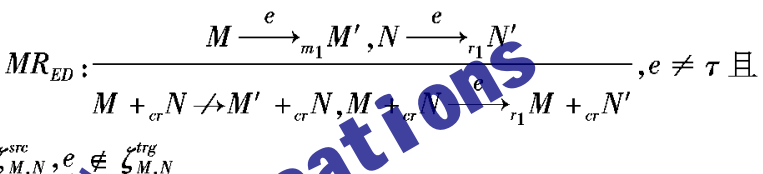

$$
\begin{aligned}
& e \in \zeta_{M, N}^{s s c}, e \notin \zeta_{M, N}^{r_{r}}
\end{aligned}
$$

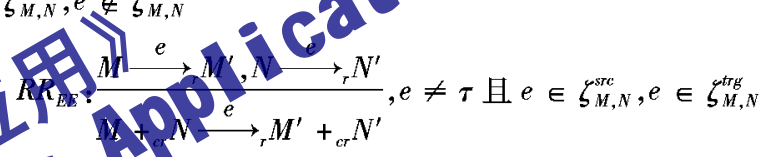

$$
\begin{aligned}
& : \frac{M \stackrel{e}{\longrightarrow}{ }_{r_{1}} M^{\prime}, N \stackrel{e}{\longrightarrow}{ }_{r_{2}} N^{\prime}}{M+{ }_{c r} N \stackrel{e}{\longrightarrow} M_{r_{1}} M^{\prime}+{ }_{c r} N, M+{ }_{c r} N \stackrel{e}{\longrightarrow} M+{ }_{r_{2}} N^{\prime}}, e \neq \tau
\end{aligned}
$$

且 $e \notin \zeta_{M, N}^{s n e}$

$$
M M_{E E}: \frac{M \stackrel{e}{\longrightarrow} M_{m}, N \stackrel{e}{\longrightarrow} N_{m}^{\prime}}{M+{ }_{c r} N \stackrel{e}{\longrightarrow} M^{\prime}+{ }_{c r} N^{\prime}}, e \neq \tau \text { 且 } e \in \zeta_{M, N}^{s s c}, e \in
$$

$\zeta_{M, N}^{\text {trg }}$

$$
\begin{aligned}
& M M_{E D}: \frac{M \stackrel{e}{\longrightarrow} m_{m_{1}} M^{\prime}, N \stackrel{e}{\longrightarrow}{ }_{m_{2}} N^{\prime}}{M+{ }_{c r} N \stackrel{e}{\longrightarrow} m_{1} M^{\prime}+{ }_{c r} N \text { 或 } M+{ }_{c r} N \stackrel{e}{\longrightarrow} m_{2} M+{ }_{c r} N^{\prime}}, \\
& e \neq \tau \text { 且, } e \in \zeta_{M, N}^{s r c}, e \notin \zeta_{M, N}^{\text {tre }} \\
& M M_{D B}: \frac{M \stackrel{e}{\longrightarrow}{ }_{m_{1}} M^{\prime}, N \stackrel{e}{\longrightarrow} m_{2} N^{\prime}}{M+{ }_{c r} N \stackrel{e}{\longrightarrow} m_{1} M^{\prime}+{ }_{c r} N, M+{ }_{c r} N \stackrel{e}{\longrightarrow} m_{m_{2}} M+{ }_{c r} N^{\prime}},
\end{aligned}
$$

$e \neq \tau$ 且 $e \notin \zeta_{M, N}^{s r c}$

下面解释上述规则, 直观上说行为模型合并就是行为模 型的组合, 就是合并后的模型中包含两个模型迁移,但是不同 的是合并过程可以对行为模型中的可能迁移用其他行为模型 的必要迁移替换或者在合并过程中删除一些可能迁移。例如 当行为模型 $M$ 在 $e$ 上是可能迁移,而模型 $N$ 在 $e$ 上是必要迁 移, 如果 $e \in \zeta_{M, N}^{s r c}, e \in \zeta_{M, N}^{\pi g}$, 根据合并规则 $R M_{E E}$ 和 $M R_{E E}$, 那么 $M+{ }_{u} N$ 中是必要迁移。以图 1 中的行为模型 $A$ 中必要迁移 $b$ ? 和 行为模型 $B$ 中的可能迁移 $b$, 在 $A$ 和 $B$ 的共同精化模型 $D$ 中是 必要迁移。如果 $e \in \zeta_{M, N}^{\mathrm{src}}, e \notin \zeta_{M, N}^{\mathrm{tr} r}$, 又根据 MTS 满足一致性, 我们可知必有一个标号为可能迁移, 在合并中我们可以将其 可能迁移删除只保留必然迁移。如果两个模型中都是可能迁 移, 那么在合并过程中可以让操作人员进行选择，也就是合 并规则中的 $M M_{E D}$ 规则。 


$$
\begin{gathered}
M D \frac{M \stackrel{e}{\longrightarrow}{ }_{m} M^{\prime}}{M+{ }_{c r} N \stackrel{e}{\longrightarrow} M_{r}{ }^{\prime}+_{c r} N} e \notin \alpha N \\
D M \frac{N \stackrel{e}{\longrightarrow}{ }_{m} N^{\prime}}{M+{ }_{c r} N \stackrel{e}{\longrightarrow} M+{ }_{c r} N^{\prime}} e \notin \alpha M
\end{gathered}
$$

如果 MTS 中的 $M$ 和 $N$ 中都不含有迁移不确定状态, 按照 隐藏操作规则所产生的共同精化就是极小共同精化。如果我 们改变 $M D$ 和 $D M$ 规则, 得到的 $M$ 和 $N$ 的共同精化就是最小共 同精化。图 1 中的行为模型 $D$ 是 $A$ 和 $B$ 的共同精化同时也是 $A$ 和 $B$ 的极小共同精化, $F$ 是模型 $A$ 和 $B$ 的最小共同精化。

\section{3 实例}

下面用一个水百控制器 ${ }^{[7]}$ 的实例来进一步说明合并规 则, 该控制器要求在高水位时, 开启洜 (switchOn), 在低水位 时关闭洜 (switchOff), 但是如果有甲醛出现 (methAppears) 必 须关闭原, 也就是说有甲醛出现时不管水位是不是高水位, 原 必须关闭。模型 $M$ 是从有甲醛出现的场景中抽取的泉关闭模 型, 模型 $N$ 是为从没有甲醛出现的场景中抽取一个洜开启行
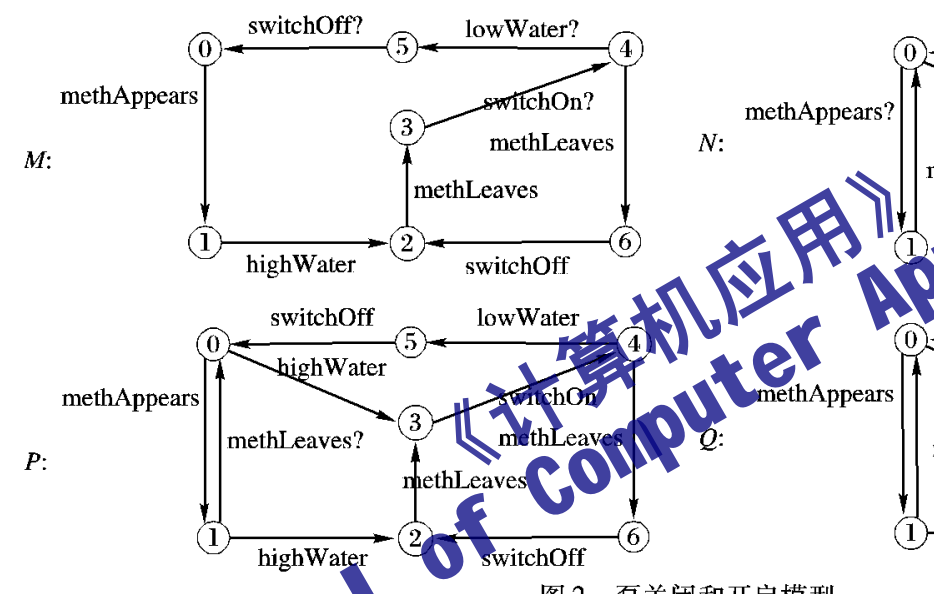

图 2 泉关闭和开启模型
为模型。要对局部的不确定行为模型 $M$ 和 $N$ 进行合并,合并成 一个完整的行为模型。用 $M=\left(S_{M}, L_{M}, \Delta_{M}^{r}, \Delta_{M}^{\nu}, s_{0_{M}}\right)$ 来表示图 2 中模型 $M$, 用 $N=\left(S_{N}, L_{N}, \Delta_{N}^{r}, \Delta_{N}^{p}, s_{0_{N}}\right)$ 表示模型 $N_{\text {。 }}$

因为 $(0$, methAppears, 1$) \in \Delta_{M}^{r},(0$, methAppears?, 1) $\in \Delta_{N}^{p}-\Delta_{N}^{r}$ 。因此根据 ${ }_{c r}$ 合并规则 $R M_{E E}$, 在共同精化模型 中事件 methAppears 从状态 0 到状态 1 的迁移是必然迁移。 同样的道理，(3, switchOn, 4)、( 4, lowWater, 5)、(5, switchOn, 0$)$ 和 $(4$, switchOn, 6 ) 在共同精化模型中都是必然 迁移。

对于事件 methLeaves 在 $M$ 中的变迁是 (2, methLeaves, 3) $\in \Delta_{M}^{r}$, 而在 $N$ 中是 $\left(1\right.$, methLeaves?,0) $\in \Delta_{N}^{p}-\Delta_{N}^{r}$ 。此事件 的源状态和目标状态都不相同, 因此在共同精化行为模型中 这两个模型不能进行合并,应该保留这两个迁移。

根据 ${ }_{c r}$ 合并规则得到 $M$ 和 $N$ 的极小共同精化 $P_{0}$ 。如果用 MCR 的 $M D$ 和 $D M$ 规则替换 $+_{c r}$ 中的 $M D$ 和 $D M$ 规则, 也就是 把 $N$ 中 $(1$, methLeaves?,0) 精化为 $(1$, methLeaves, 0$)$ 得到 $M$ 和 $N$ 的最小共同精化 $Q$ 。

$N$ :
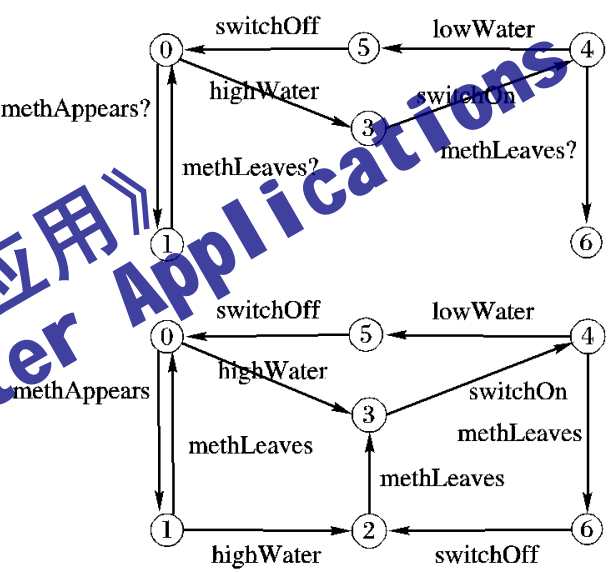

3 结语

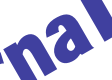

models from scenarios $[\mathrm{J}]$. IEEE Transactions on Software Engineering, 2003, 29(2): $99-115$.

文中采用模态迁楮系统描述系统行为模型, 通过对行为 模型精化关系的分析提出了精化模型, 使用隐藏操作解决了 精化定义中要求有相同通信字母表的问题,并在此基础上给 出两个行为模型的共同精化模型, 根据共同精化模型的精化 程度定义出极小共同精化模型和最小共同精化模型。针对现 有合并算法的局限性, 给出了源状态一致标号集和目标状态 一致标号集来解决合并过程中总是假定事件的源状态和目标 状态一致的问题,并结合精化关系给出了新的合并规则。使 用行为模型的一致性和迁移不确定状态规定被合并行为模型 要满足的条件,行为模型的一致性确保被合并的两个模型之 间不存在互相矛盾的迁移关系, 行为模型不含有迁移不确定 状态保证了行为模型的每个状态通过同一事件迁移到唯一状 态。本文主要解决了局部行为模型的合并问题, 对于能够进 行合并的 MTS 模型只提到要满足一致性和不能含有迁移不 确定状态, 接下来我们将进一步研究局部行为模型能够进行 合并要满足的条件, 并希望给出 MTS 模型一致性检验的 方法。

\section{参考文献：}

[1] UCHITEL S, KRAMER J, MAGEE J. Synthesis of behavioural

[2] DAMS D. Abstract interpretation and partition refinement for model checking [D]. Eindhoven, Netherlands: Eindhoven University of Technology, 1996.

[3] CHECHIK M, DEVEREUX B, EASTERBROOK S, et al. Multivalued symbolic model-checking $\mathrm{J}]$. ACM Transactions on Software Engineering and Methodology, 2003, 12(4): 1 -38.

[4] BRUNET G. A characterization of merging partial behavioural mod$\operatorname{els}[\mathrm{EB} / \mathrm{OL}]$. [ $2009-04-15]$. http: //www. cs. toronto. edu/ chechik/pubs/GregBrunetMSThesis. pdf.

[5] UCHITEL S , CHECHIK M . Merging partial behavioural models [C]// Proceedings of 12th ACM SIGSOFT International Symposium on Foundations of Software Engineering. New York: ACM, 2004: 43 -52 .

[6] LARSEN K G, STEFFEN B, WEISE C. A constraint oriented proof methodology based on modal transition systems[C]// Proceedings of the 1st Intermational Workshop on Tools and Algorithms for Construction and Analysis of Systems. London: Springer-Verlag, 1995: 17 40 .

[7] DAMAS G, LAMBEAU B, DUPONT P. Generating annotated behavior models from end-user scenarios $[\mathrm{J}]$. IEEE Trnsactions on Software Engineering, 2005,31(12): 1056 - 1073 . 